\title{
ON SOME PROBLEMS OF SH WAVE PROPAGATION IN INHOMOGENEOUS ELASTIC BODIES
}

\author{
Sebastian Kowalczyk, StanisŁaw J. Matysiak \\ University of Warsaw, Faculty of Geology, Institute of Hydrogeology and Engineering Geology, Warszawa, Poland \\ e-mail: s.j.matysiak@uw.edu.pl \\ DARIUsz M. Perkowski \\ Bialystok University of Technology, Faculty of Mechanical Engineering, Bialystok, Poland
}

\begin{abstract}
The paper deals with the propagation of shear horizontal ( $\mathrm{SH}$ ) waves in an nonhomogeneous elastic half-space composed of a layer whose shear modulus and mass density have a power dependence on the distance from the lower plane and the periodically stratified half-space. The equation which relates the wave speed to the wave-number and functions of the shear modulus and mass density is derived. The wave velocity is analyzed numerically. Especially, the influence of mechanical properties of the coating layer and the stratified foundation on the wave velocity is presented in the form of figures.
\end{abstract}

Keywords: displacement, stresses, SH wave, shear modulus, stratified foundation

\section{Introduction}

The phenomena of wave propagations through the Earth is useful in investigating the internal Earth structure, and it can be helpful in explorations of various materials beneath the Earth's surface. It is well known that the Earth is not perfectly homogeneous and some forms of inhomogeneity exist. Many rocks and soils are stratified and clearly pice-wise homogeneous. Some layers are characterized by mechanical parameters with continuous changing in spatial directions (called as functionally graded materials). The problems of modeling of wave propagations in inhomogeneous elastic bodies play a very important role in applied geophysics civil and mechanical engineering (space structures, fusion reactors). The list of references connected with the problems of wave propagations in inhomogeneous elastic bodies is rather very large (for instance monographsby by Birykov et al. (1995), Brekhovskikh (1960), Kennet (1983), Nayfeh (1995); papers by Achenbach and Balogun (2010), Alenitsyn (1964), Alshits and Maugin (2005), Cerveny et al. (1982), Destrade (2007), Shuvalov et al. (2008), Vrettos (1990)). Achenbach and Balogun (2010) dealt with the propagation of anti-plane shear waves in an elastic half-space whose shear modulus and mass density had an arbitrary dependence on the distance from the boundary plane. Alenitsyn (1964) considered the problem of Rayleigh waves in a nonhomogenous elastic slab. Alshits and Maugin (2005) developed a theory for the elastic wave propagation in an arbitrary layered plane (piece-wise homogenous or continuously inhomogeneous). The description was based on the transfer matrix approach. The Gaussian beam method of the solution of wave propagation problems in inhomogeneous bodies was applied by Cerveny et al. (1982). The seismic Rayleigh waves in an orthotropic elastic half-space with an exponentially graded properties were considered by Destrade (2007). Shuvalov et al. (2008) presented some analysis of the problem of shear horizontal waves in transversely inhomogeneous plates. Surface harmonic vibrations of soil deposits with variable shear modulus were analysed by Vrettos (1990).

The problem of SH-wave propagation in anisotropic inhomogeneous layer with directional rigidities and density of mass changing as a power function was investigated by Upadhyay and 
Gupta (1972). The authors assumed that the outer layer was fixed to an isotropic homogeneous elastic half-space or to the rigid substrate.

The present paper is concerned with the case of a shear horizontal $(\mathrm{SH})$ wave in an inhomogeneous elastic layer which is assumed to be ideally fixed to a periodically stratified elastic half-space, and the upper boundary plane is free of loadings. The considered layer is characterized by the shear modulus and mass density in the form of power functions of the distance from the lower boundary plane. The substrate is assumed to be composed of periodically repeated two-layered laminae parallel to the boundary plane. Each component of the lamina is a homogeneous and isotropic body. The assumptions connected with the ideal bonding of the components on interfaces lead to a complicated boundary value problem within the framework of the classical theory of elasticity. For this reason, the classic idea is the use of the approximate procedure to replace the heterogeneous medium by an equivalent homogenized model, which gives the average behavior at the macroscopic scale. One of them is the homogenized model with microlocal parameters presented by Matysiak and Woźniak (1987, 1988). This model is derived by using the methods of the nonstandard analysis and taking into account the effects due to the periodic structure of the body. The governing equations of the model are formulated in terms of the unknown macro-displacements and certain extra unknowns being referred to as microlocal parameters. They are described by a relatively simple form of the equations satisfying the conditions of perfect interfacial bonding of constituents. The homogenized model has been successfully applied to a series of problems of the linear elasticity and thermoelasticity (problems of cracks, cavities, inclusions, contact problems, wave propagations), which it was partially resumed in (Matysiak, 1996; Woźniak and Woźniak, 1995). It should be underlined that the homogenization approach has been noticed to produce good physical results, at the same time being rather simple in mathematical aspects (Kulchytsky-Zhyhailo and Kołodziejczyk, 2007; Kulchytsky-Zhyhailo and Matysiak, 2005, 2006; Kulchytsky-Zhyhailo et al., 2006). The wave problems in a periodically layered elastic half-space were investigated by Bielski and Matysiak (1992), Matysiak et al. (2009). The same dependence of the shear modulus is taken into account in many papers (see for instance Calladine and Greenwood, 1978; Wang et al., 2003)). The same dependence of the shear modulus of the coating layer is considered in the present paper. The distributions of displacements and stresses in an inhomogeneous incompressible elastic half-space caused by line and point loads are considered in (Cerveny et al., 1982). The propagation of surface waves in a linear-elastic, isotropic, compressible half-space with constant mass density and Poisson's ratio and shear modulus varying with depth is considered in (Vrettos, 1990). The useful list of dependence forms for elastic modulus is presented by Wang et al. (2003).

\section{Formulation and solution of the problem}

Consider the problem of shear waves propagation in an elastic nonhomogeneous layer and periodically layered half-space. Let $\left(x_{1}, x_{2}, x_{3}\right)$ denote the Cartesian coordinate system such that the layer occupies the region $x_{1} \in R, 0 \leqslant x_{2} \leqslant H, x_{3} \in R$, where $H>0$ is constant thickness of the FGM body, Fig. 1.

Let the upper boundary plane $x_{2}=H$ be free of loadings, and the layer is ideally fixed to the periodically two-layered half-space in the plane $x_{2}=0$. Let the stratified half-space be composed of periodically repeated fundamental laminae with thickness $\delta$, which include two homogeneous isotropic sub-layers denoted by 1 and 2 with thicknesses $\delta_{j}, j=1,2$, and $\delta=\delta_{1}+\delta_{2}$. Let $\mu_{j}, \rho_{j}$, $j=1,2$ be the shear modulus and mass densities of the subsequent constituents of the composite half-space. Herein and in the sequel, all quantities (material components, stresses) pertaining to sub-layer 1 and 2 will be labeled by the index $j$ taking values 1 and 2 , respectively. The 


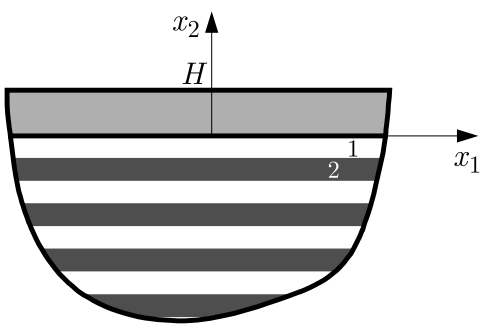

Fig. 1. Scheme of the considered $\mathrm{SH}$ problem

considerations are limited to the anti-plane harmonic wave propagation in the $0 x_{1}$ direction. Let $\mathbf{u}\left(x_{1}, x_{2}, t\right)=\left(0,0, u_{3}\left(x_{1}, x_{2}, t\right)\right)$ be the displacement vector, where $t$ denotes time. The shear modulus $\mu$ and mass density $\rho$ of the upper layer are assumed the same as in (Upadhyay and Gupta, 1972), namely

$$
\mu=\mu_{0}\left(1+\alpha x_{2}\right)^{p} \quad \rho=\rho_{0}\left(1+\alpha x_{2}\right)^{p}
$$

where $\mu_{0}, \rho_{0}, \alpha, p$ are given constants.

The non-zero stress components $\sigma_{13}$ and $\sigma_{23}$ in the coating layer are expressed in the form

$$
\begin{aligned}
& \sigma_{13}\left(x_{1}, x_{2}, t\right)=\mu_{0}\left(1+\alpha x_{2}\right)^{p} \frac{\partial u_{3}\left(x_{1}, x_{2}, t\right)}{\partial x_{1}} \\
& \sigma_{23}\left(x_{1}, x_{2}, t\right)=\mu_{0}\left(1+\alpha x_{2}\right)^{p} \frac{\partial u_{3}\left(x_{1}, x_{2}, t\right)}{\partial x_{2}}
\end{aligned}
$$

The anti-plane wave motion is governed by the following equation

$$
\frac{\partial \sigma_{13}}{\partial x_{1}}+\frac{\partial \sigma_{23}}{\partial x_{2}}=\rho_{0}\left(1+\alpha x_{2}\right)^{p} \frac{\partial^{2} u_{3}}{\partial t^{2}} \quad x_{1} \in R \quad 0<x_{2}<H
$$

where the body forces are omitted. From equations (2.2) and (2.3), it follows that

$$
\frac{\partial^{2} u_{3}}{\partial x_{1}^{2}}+\frac{\alpha p}{1+\alpha x_{2}} \frac{\partial u_{3}}{\partial x_{2}}+\frac{\partial^{2} u_{3}}{\partial x_{2}^{2}}=\frac{\rho_{0}}{\mu_{0}} \frac{\partial^{2} u_{3}}{\partial t^{2}} \quad x_{1} \in R \quad 0<x_{2}<H \quad t \in R
$$

To determine the displacement and stresses in the periodically layered half-space $x_{2}<0$, the homogenized model with microlocal parameters (Bielski and Matysiak, 1992; Kulchytsky-Zhyhailo and Kołodziejczyk, 2007; Kulchytsky-Zhyhailo and Matysiak, 2005, 2006; Kulchytsky-Zhyhailo et al., 2006; Matysiak et al., 2009; Matysiak and Woźniak, 1987, 1988) is applied. Here only a brief outline of the governing equations for the case of anti-plane state of strain will be presented. The homogenized procedure presented by Matysiak and Woźniak $(1987,1988)$ is based on theorems of the nonstandard analysis and some physical assumptions, which leads, in the case of anti-plane state of strain, to the following approximations

$$
\begin{aligned}
& u_{3}\left(x_{1}, x_{2}, t\right)=w_{3}\left(x_{1}, x_{2}, t\right)+h\left(x_{2}\right) q_{3}\left(x_{1}, x_{2}, t\right) \approx w_{3}\left(x_{1}, x_{2}, t\right) \\
& \frac{\partial u_{3}\left(x_{1}, x_{2}, t\right)}{\partial x_{1}} \approx \frac{\partial w_{3}\left(x_{1}, x_{2}, t\right)}{\partial x_{1}} \quad \frac{\partial u_{3}\left(x_{1}, x_{2}, t\right)}{\partial t} \approx \frac{\partial w_{3}\left(x_{1}, x_{2}, t\right)}{\partial t} \quad \\
& \frac{\partial u_{3}\left(x_{1}, x_{2}, t\right)}{\partial x_{2}} \approx \frac{\partial w_{3}\left(x_{1}, x_{2}, t\right)}{\partial x_{2}}+h^{\prime}\left(x_{2}\right) q_{3}\left(x_{1}, x_{2}, t\right)
\end{aligned}
$$

where $w_{3}, q_{3}$ are unknowns called macro-displacement and microlocal parameters, respectively. The function $h$ (called the shape function) is given in the form

$$
\begin{array}{ll}
h\left(x_{2}\right)= \begin{cases}x_{2}-\frac{1}{2} \delta_{1} & \text { for } \quad 0 \leqslant x_{2} \leqslant \delta_{1} \\
-\frac{\eta x_{2}}{1-\eta}-\frac{1}{2} \delta_{1}+\frac{\delta_{1}}{1-\eta} & \text { for } \quad \delta_{1} \leqslant x_{2} \leqslant \delta\end{cases} \\
h\left(x_{2}+\delta\right)=h\left(x_{2}\right) &
\end{array}
$$


and

$$
\eta=\frac{\delta_{1}}{\delta}
$$

Since $\left|h\left(x_{2}\right)\right|<\delta$ for every $x_{2} \in R$, then for small $\delta$ the terms with $h$ in equations (2.5) are small and are neglected. However, the derivative $h^{\prime}$ is not small and the terms involving $h^{\prime}$ cannot be neglected. The form of the shape function $h$ given in (2.6) secures the fulfilment of the conditions of ideal bonding on the composite interfaces. The homogenized model presented by Matysiak and Woźniak $(1987,1988)$ in the case of anti-plane state of strain leads to the following equations for the unknowns $w_{3}$ and $q_{3}$

$$
\widetilde{\mu}\left(\frac{\partial^{2} w_{3}}{\partial x_{1}^{2}}+\frac{\partial^{2} w_{3}}{\partial x_{2}^{2}}\right)+[\mu] \frac{\partial q_{3}}{\partial x_{2}}=\widetilde{\rho} \frac{\partial^{2} w_{3}}{\partial t^{2}} \quad \widehat{\mu} q_{3}+[\mu] \frac{\partial w_{3}}{\partial x_{2}}=0
$$

where

$$
\begin{array}{ll}
\widetilde{\rho}=\eta \rho_{1}+(1-\eta) \rho_{2} & \widetilde{\mu}=\eta \mu_{1}+(1-\eta) \mu_{2} \\
{[\mu]=\eta\left(\mu_{1}-\mu_{2}\right)} & \widehat{\mu}=\eta \mu_{1}+\frac{\eta^{2} \mu_{2}}{1-\eta}
\end{array}
$$

The non-zero stress components $\sigma_{13}^{(j)}, \sigma_{23}^{(j)}, j=1,2$ in the layer of $j$-th kind are expressed in the form

$$
\sigma_{13}^{(j)}=\mu_{j} \frac{\partial w_{3}}{\partial x_{1}} \quad \sigma_{23}^{(j)}=\mu_{j}\left(\frac{\partial w_{3}}{\partial x_{2}}+h^{\prime}\left(x_{2}\right) q_{3}\right)
$$

Eliminating the microlocal parameter $q_{3}$ from $(2.8)_{1}$ (2.10) by using $(2.8)_{2}$, leads to the equations

$$
\widetilde{\mu} \frac{\partial^{2} w_{3}}{\partial x_{1}^{2}}+C \frac{\partial^{2} w_{3}}{\partial x_{2}^{2}}=\widetilde{\rho} \frac{\partial^{2} w_{3}}{\partial t^{2}}
$$

and

$$
\sigma_{13}^{(j)}=\mu_{j} \frac{\partial w_{3}}{\partial x_{1}} \quad \sigma_{23}^{(j)}=C \frac{\partial w_{3}}{\partial x_{2}} \quad j=1,2
$$

where

$$
C=\widetilde{\mu}-\frac{[\mu]^{2}}{\widehat{\mu}}=\frac{\mu_{1} \mu_{2}}{(1-\eta) \mu_{1}+\eta \mu_{2}}>0
$$

The following boundary conditions are taken into consideration:

a) on the upper boundary of the FGM layer

$$
\sigma_{23}\left(x_{1}, H, t\right)=0 \quad x_{1} \in R \quad t \in R
$$

b) on the interface $x_{2}=0$ between the FGM layer and the periodically stratified half-space

$$
\begin{aligned}
& u_{3}\left(x_{1}, 0^{+}, t\right)=w_{3}\left(x_{1}, 0^{-}, t\right) \quad \sigma_{23}\left(x_{1}, 0^{+}, t\right)=\sigma_{23}^{(1)}\left(x_{1}, 0^{-}, t\right) \\
& x_{1} \in R \quad t \in R
\end{aligned}
$$

c) the regularity condition at infinity

$$
\lim _{x_{2} \rightarrow-\infty} w_{3}\left(x_{1}, x_{2}, t\right)=0
$$


Let us consider a SH wave solution of the form

$$
u_{3}\left(x_{1}, x_{2}, t\right)=U_{3}\left(x_{2}\right) \mathrm{e}^{\mathrm{i} k\left(x_{1}-c t\right)} \quad w_{3}\left(x_{1}, x_{2}, t\right)=W_{3}\left(x_{2}\right) \mathrm{e}^{\mathrm{i} k\left(x_{1}-c t\right)}
$$

where $\mathrm{i}=\sqrt{-1}, U_{3}$ and $W_{3}$ are unknown amplitude of displacement in the outer layer and the periodically layered half-space, respectively, and $k$ and $c$ are the wave number and the phase velocity, respectively. By using equations (2.4) and (2.11) and (2.17), an ordinary differential equation are obtained

$$
\frac{d^{2} U_{3}\left(x_{2}\right)}{d x_{2}^{2}}+\frac{\alpha p}{1+\alpha x_{2}} \frac{d U_{3}\left(x_{2}\right)}{d x_{2}}+k^{2}\left(\frac{c^{2}}{c_{0}^{2}}-1\right) U_{3}\left(x_{2}\right)=0 \quad 0<x_{2}<H
$$

and

$$
\frac{d^{2} W_{3}\left(x_{2}\right)}{d x_{2}^{2}}+\frac{k^{2}}{C}\left(\widetilde{\rho} c^{2}-\widetilde{\mu}\right) W_{3}\left(x_{2}\right)=0 \quad x_{2}<0
$$

where

$$
c_{0}^{2}=\frac{\mu_{0}}{\rho_{0}}
$$

The ordinary differential equation of the second order with variable coefficients (2.18) belongs to well-known type (Kamke, 1976, p. 401). Its general solution has the form

$$
U_{3}\left(x_{2}\right)=\left(1+\alpha x_{2}\right)^{\frac{1-p}{2}}\left[A_{1} J_{\frac{|1-p|}{2}}\left(q\left(\frac{1}{\alpha}+x_{2}\right)\right)+A_{2} Y_{\frac{|1-p|}{2}}\left(q\left(\frac{1}{\alpha}+x_{2}\right)\right)\right] \quad 0<x_{2}<H
$$

where

$$
q^{2}=k^{2}\left(\frac{c^{2}}{c_{0}^{2}}-1\right)
$$

on the assumption that $c>c_{0}$, and $A_{1}, A_{2}$ are unknown constants, which should be determined from boundary conditions (2.5), and $J_{|1-p| / 2}(\cdot), Y_{|1-p| / 2}(\cdot)$ are Bessel functions. Equations (2.19) and (2.17) with condition (2.16) lead to the following solution

$$
W_{3}\left(x_{2}\right)=A_{3} \exp \left(\beta x_{2}\right) \quad x_{2}<0 \quad \beta^{2}=\frac{k^{2} \widetilde{\mu}}{C}\left(1-\frac{c^{2}}{\widetilde{c}^{2}}\right) \quad \widetilde{c}^{2}=\frac{\widetilde{\mu}}{\widetilde{\rho}}
$$

on the assumption that $c<\widetilde{c}$ and $A_{3}$ is an unknown constant. The constant $A_{1}, A_{2}, A_{3}$ should be calculated from boundary conditions (2.14) and (2.15).

The further analysis needs to take into consideration two cases: $p \leqslant 1$ and $p>1$.

\section{Case 1}

Consider that

$$
p \leqslant 1 \quad \text { so } \quad|1-p|=1-p
$$

To determine the stress component $\sigma_{23}$, the following differential relations for the Bessel functions should be applied (Lebiediev, 1957)

$$
\frac{d z^{\nu} J_{\nu}(z)}{d z}=z^{\nu} J_{\nu-1}(z) \quad \frac{d z^{\nu} Y_{\nu}(z)}{d z}=z^{\nu} Y_{\nu-1}(z)
$$


Bearing in mind equations $(2.2),(2.17),(2.21)$ and (2.24), it follows that the stress component $\sigma_{23}$ is expressed in the form

$$
\sigma_{23}\left(x_{1}, x_{2}, t\right)=q \mu_{0}\left(1+\alpha x_{2}\right)^{\frac{1+p}{2}}\left[A_{1} J_{\frac{-1-p}{2}}\left(q\left(\frac{1}{\alpha}+x_{2}\right)\right)+A_{2} Y_{\frac{-1-p}{2}}\left(q\left(\frac{1}{\alpha}+x_{2}\right)\right)\right] \mathrm{e}^{\mathrm{i} k\left(x_{1}-c t\right)}
$$

where $0<x_{2}<H$.

From boundary condition (2.14) and conditions of continuity (2.15) as well as equations (2.26), (2.17), (2.23), (2.12), (2.21), the following algebraic equations for the unknowns $A_{1}, A_{2}$, $A_{3}$ are obtained

$$
\begin{aligned}
& A_{1} J_{\frac{-1-p}{2}}\left(q\left(\frac{1}{\alpha}+H\right)\right)+A_{2} Y_{\frac{-1-p}{2}}\left(q\left(\frac{1}{\alpha}+H\right)\right)=0 \\
& A_{1} J_{\frac{1-p}{2}}\left(\frac{q}{\alpha}\right)+A_{2} Y_{\frac{1-p}{2}}\left(\frac{q}{\alpha}\right)=A_{3} \\
& \mu_{0} q\left[A_{1} J_{\frac{-1-p}{2}}\left(\frac{q}{\alpha}\right)+A_{2} Y_{\frac{-1-p}{2}}\left(\frac{q}{\alpha}\right)\right]=C \beta A_{3}
\end{aligned}
$$

Eliminating $A_{3}$ from the system of equations (2.27), it follows that

$$
\begin{aligned}
& A_{1} J_{\frac{-1-p}{2}}\left(q\left(\frac{1}{\alpha}+H\right)\right)+A_{2} Y_{\frac{-1-p}{2}}\left(q\left(\frac{1}{\alpha}+H\right)\right)=0 \\
& A_{1}\left[\mu_{0} q J_{\frac{-1-p}{2}}\left(\frac{q}{\alpha}\right)-C \beta J_{\frac{1-p}{2}}\left(\frac{q}{\alpha}\right)\right]+A_{2}\left[\mu_{0} q Y_{\frac{-1-p}{2}}\left(\frac{q}{\alpha}\right)-C \beta Y_{\frac{1-p}{2}}\left(\frac{q}{\alpha}\right)\right]=0
\end{aligned}
$$

The system of algebraic equations (2.28) has a non-zero solution under the following condition

$$
\begin{aligned}
& J_{\frac{-1-p}{2}}\left(q\left(\frac{1}{\alpha}+H\right)\right)\left[\mu_{0} q Y_{\frac{-1-p}{2}}\left(\frac{q}{\alpha}\right)-C \beta Y_{\frac{1-p}{2}}\left(\frac{q}{\alpha}\right)\right] \\
& \quad-Y_{\frac{-1-p}{2}}\left(q\left(\frac{1}{\alpha}+H\right)\right)\left[\mu_{0} q J_{\frac{-1-p}{2}}\left(\frac{q}{\alpha}\right)-C \beta J_{\frac{1-p}{2}}\left(\frac{q}{\alpha}\right)\right]=0
\end{aligned}
$$

Equation (2.29) will be solved numerically.

\section{Case 2}

Consider now that

$$
p>1 \quad \text { so } \quad|1-p|=p-1
$$

To determine the stress component $\sigma_{23}$, the following differential relations for the Bessel functions should be applied (Lebiediev, 1957)

$$
\frac{d z^{-\nu} J_{\nu}(z)}{d z}=-z^{-\nu} J_{\nu+1}(z) \quad \frac{d z^{-\nu} Y_{\nu}(z)}{d z}=-z^{-\nu} Y_{\nu+1}(z)
$$

Bearing in mind equations (2.2), (2.17), (2.21) and (2.31), it follows that the stress component $\sigma_{23}$ is expressed in the form

$$
\sigma_{23}\left(x_{1}, x_{2}, t\right)=-q \mu_{0}\left(1+\alpha x_{2}\right)^{\frac{1+p}{2}}\left[A_{1} J_{\frac{1+p}{2}}\left(q\left(\frac{1}{\alpha}+x_{2}\right)\right)+A_{2} Y_{\frac{1+p}{2}}\left(q\left(\frac{1}{\alpha}+x_{2}\right)\right)\right] \mathrm{e}^{\mathrm{i} k\left(x_{1}-c t\right)}
$$

where $0<x_{2}<H$. 
From boundary conditions (2.14) and (2.15) and equations (2.32), (2.17), (2.23), (2.12), (2.21), the following linear algebraic equations for the unknowns $A_{1}, A_{2}, A_{3}$ are obtained

$$
\begin{aligned}
& A_{1} J_{\frac{p+1}{2}}\left(\frac{q}{\alpha}(1+\alpha H)\right)+A_{2} Y_{\frac{p+1}{2}}\left(\frac{q}{\alpha}(1+\alpha H)\right)=0 \\
& A_{1} J_{\frac{p+1}{2}}\left(\frac{q}{\alpha}\right)+A_{2} Y_{\frac{p+1}{2}}\left(\frac{q}{\alpha}\right)=A_{3} \\
& -\mu_{0} q\left[A_{1} J_{\frac{1+p}{2}}\left(\frac{q}{\alpha}\right)+A_{2} Y_{\frac{1+p}{2}}\left(\frac{q}{\alpha}\right)\right]=C \beta A_{3}
\end{aligned}
$$

Eliminating $A_{3}$ from the system of equations (2.33), it follows that

$$
\begin{aligned}
& A_{1} J_{\frac{p+1}{2}}\left(\frac{q}{\alpha}(1+\alpha H)\right)+A_{2} Y_{\frac{p+1}{2}}\left(\frac{q}{\alpha}(1+\alpha H)\right)=0 \\
& A_{1}\left[\mu_{0} q J_{\frac{p+1}{2}}\left(\frac{q}{\alpha}\right)+C \beta J_{\frac{p-1}{2}}\left(\frac{q}{\alpha}\right)\right]+A_{2}\left[\mu_{0} q Y_{\frac{p+1}{2}}\left(\frac{q}{\alpha}\right)+C \beta Y_{\frac{p-1}{2}}\left(\frac{q}{\alpha}\right)\right]=0
\end{aligned}
$$

The system of algebraic equations (2.34) has non-zero solutions under the following condition

$$
\begin{aligned}
& J_{\frac{p+1}{2}}\left(\frac{q}{\alpha}(1+\alpha H)\right)\left[\mu_{0} q Y_{\frac{p+1}{2}}\left(\frac{q}{\alpha}\right)+C \beta Y_{\frac{p-1}{2}}\left(\frac{q}{\alpha}\right)\right] \\
& \quad-Y_{\frac{p+1}{2}}\left(\frac{q}{\alpha}(1+\alpha H)\right)\left[\mu_{0} q J_{\frac{p+1}{2}}\left(\frac{q}{\alpha}\right)+C \beta J_{\frac{p-1}{2}}\left(\frac{q}{\alpha}\right)\right]=0
\end{aligned}
$$

Equation (2.35) will be solved numerically.

\section{Numerical results}

Equations (2.29) and (2.35) will be solved numerically applying the bisection method. For this aim, the following notations are introduced

$$
\psi=\frac{c^{2}}{c_{0}^{2}} \quad \widehat{C}=\frac{C \beta}{\mu_{0}}
$$

\section{Case 1}

For $p \leqslant 1$ from (2.28) and (3.1), it follows that

$$
\begin{aligned}
& J_{\frac{-1-p}{2}}\left(\frac{k}{\alpha} \sqrt{\psi-1}(1+\alpha H)\right)\left[k \sqrt{\psi-1} Y_{\frac{-1-p}{2}}\left(\frac{k}{\alpha} \sqrt{\psi-1}\right)-\widehat{C} Y_{\frac{1-p}{2}}\left(\frac{k}{\alpha} \sqrt{\psi-1}\right)\right] \\
& -Y_{\frac{-1-p}{2}}\left(\frac{k}{\alpha} \sqrt{\psi-1}(1+\alpha H)\right)\left[k \sqrt{\psi-1} J_{\frac{-1-p}{2}}\left(\frac{k}{\alpha} \sqrt{\psi-1}\right)-\widehat{C} J_{\frac{1-p}{2}}\left(\frac{k}{\alpha} \sqrt{\psi-1}\right)\right]=0
\end{aligned}
$$

\section{Case 2}

For $p>1$ from (3.1) and (2.35), it follows that

$$
\begin{aligned}
& J_{\frac{p+1}{2}}\left(\frac{k}{\alpha} \sqrt{\psi-1}(1+\alpha H)\right)\left[k \sqrt{\psi-1} Y_{\frac{p+1}{2}}\left(\frac{k}{\alpha} \sqrt{\psi-1}\right)+\widehat{C} Y_{\frac{p-1}{2}}\left(\frac{k}{\alpha} \sqrt{\psi-1}\right)\right] \\
& -Y_{\frac{p+1}{2}}\left(\frac{k}{\alpha} \sqrt{\psi-1}(1+\alpha H)\right)\left[k \sqrt{\psi-1} J_{\frac{p+1}{2}}\left(\frac{k}{\alpha} \sqrt{\psi-1}\right)+\widehat{C} J_{\frac{p-1}{2}}\left(\frac{k}{\alpha} \sqrt{\psi-1}\right)\right]=0
\end{aligned}
$$

The obtained numerical results for the dimensionless ratio $\psi=c^{2} / c_{0}^{2}$ are presented in the form of figures. Figure $2 \mathrm{a}$ presents the ratio $\psi$ as a function of the parameter $p$ for three cases of $k H=1,2,4$, parameters $\eta=0.5, \alpha=0.05$ and ratios $\mu_{1} / \mu_{2}=4, \mu_{1} / \mu_{0}=2, \rho_{1} / \rho_{0}=2$. For $\alpha=0.05$ and a small values of $H$ being the thickness of the FGM layer it follows form equation 
(a)

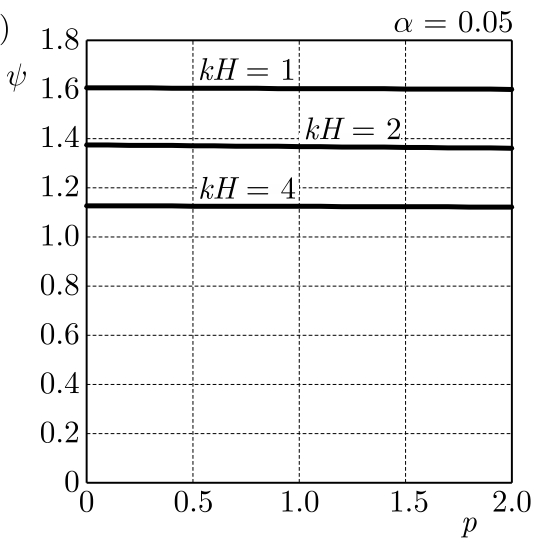

(b)

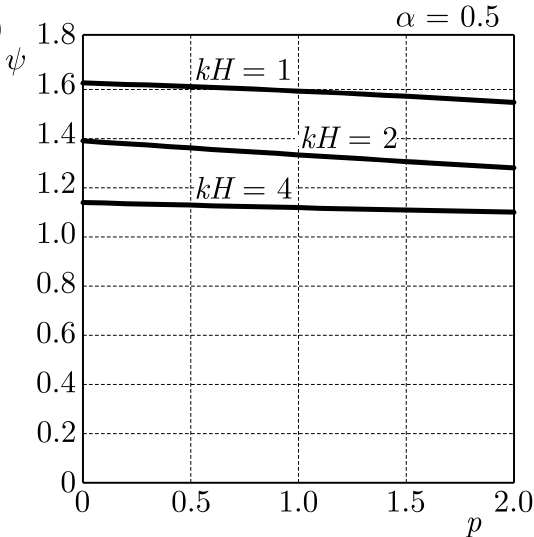

Fig. 2. The distribution of the parameter $\psi=c^{2} / c_{0}^{2}$ as a function of the parameter $p$ for $k H=1,2,4$, $\eta=0.5, \mu_{1} / \mu_{2}=4, \mu_{1} / \mu_{0}=2, \rho_{1} / \rho_{2}=2, \rho_{1} / \rho_{0}=2 ;$ (a) $\alpha=0.05$, (b) $\alpha=0.5$

(2.1) that the coating layer is almost homogeneous for all values of $p$. For this reason, the values of the ratio $\psi$ are almost constant. A different case is presented in Fig. 2b, where the same values of the parameters as in Fig. 2a are taken into account without the parameter $\alpha=0.5$. A weak influence of the nonhomogeneity of the coating layer on the wave speed $\psi=c^{2} / c_{0}^{2}$ can be noticed.

Figure 3a presents the distributions of $\psi$ as functions of $k H$ for $\eta=0.5, \mu_{1} / \mu_{0}=2, \rho_{1} / \rho_{0}=2$, $\alpha=0.05, p=0.5$ and three cases of values of the ratios: $1-\mu_{1} / \mu_{2}=4,2-\mu_{1} / \mu_{2}=6$, $3-\mu_{1} / \mu_{2}=8$. This figure shows that the influence of different features of the sub-layers being components of the considered foundation on the wave speed $\psi=c^{2} / c_{0}^{2}$ is rather small.

The distributions of $\psi$ as a function of the ratio $\mu_{1} / \mu_{2}=\rho_{1} / \rho_{2}$ for four cases of values $p=0,0.5,1,2$ and $\eta=0.5, \mu_{1} / \mu_{0}=2, \rho_{1} / \rho_{0}=2, \alpha=0.05, k H=1$ are presented in Fig. 3b. The curve numbered by 1 (Fig. 3b) shows the dependence of the ratio $\psi$ for $p=0$, so it is the homogenous coating layer and the periodically layered foundation. It can be observed that values of $\psi$ decrease together with an increase in the parameter $p$.

(a)

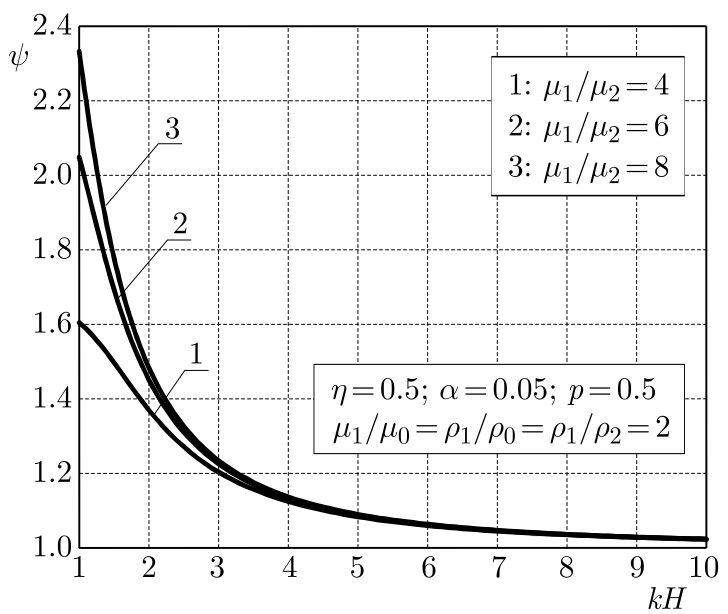

(b)

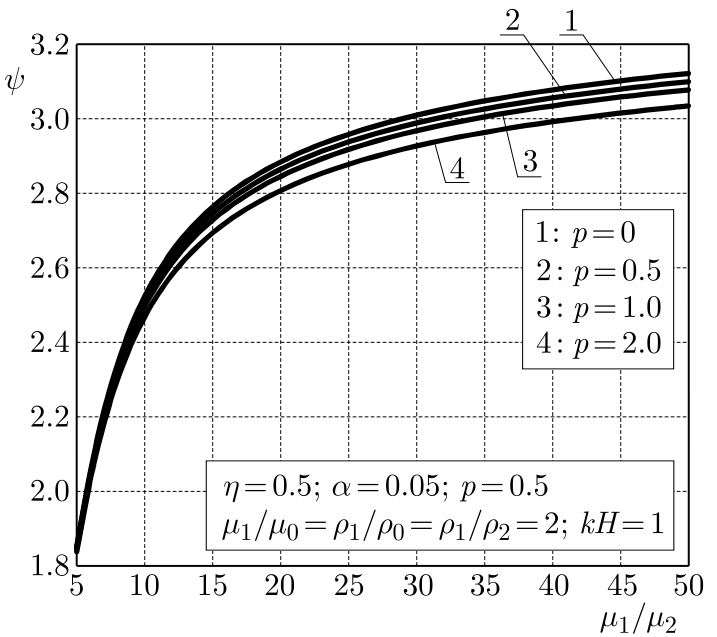

Fig. 3. The distributions of $\psi=c^{2} / c_{0}^{2}$ : (a) as a function of $k H$ for $1: \mu_{1} / \mu_{2}=4,2: \mu_{1} / \mu_{2}=6$, 3: $\mu_{1} / \mu_{2}=8 ;$ (b) as a function of $\mu_{1} / \mu_{2}$

Figure 4a shows the distributions of the ratio $\psi$ as a function of $\mu_{1} / \mu_{0}$ for four cases of the ratio $\mu_{1} / \mu_{2}=4,6,8,10$ and $\eta=0.5, \alpha=0.05, \rho_{1} / \rho_{0}=\rho_{1} / r h o_{2}=2, p=0.5, k H=1$. The curve numbered by 1 presents the smallest values of $\psi$ for all the considered nonhomogenities of the periodically layered foundation. 
The distributions of the ratio $\psi$ as a function of the parameter $\eta$ for $\alpha=0.05$, $\mu_{1} / \mu_{0}=\rho_{1} / \rho_{0}=\rho_{1} / \rho_{2}=2, p=0.5, k H=1$ and for cases of the ratio $\mu_{1} / \mu_{2}=4,6,8,10$ are given in Fig. 4b. It can be observed that for $\eta \rightarrow 1$ all curves numbered by $1,2,3$ and 4 tend to the same point.

(a)

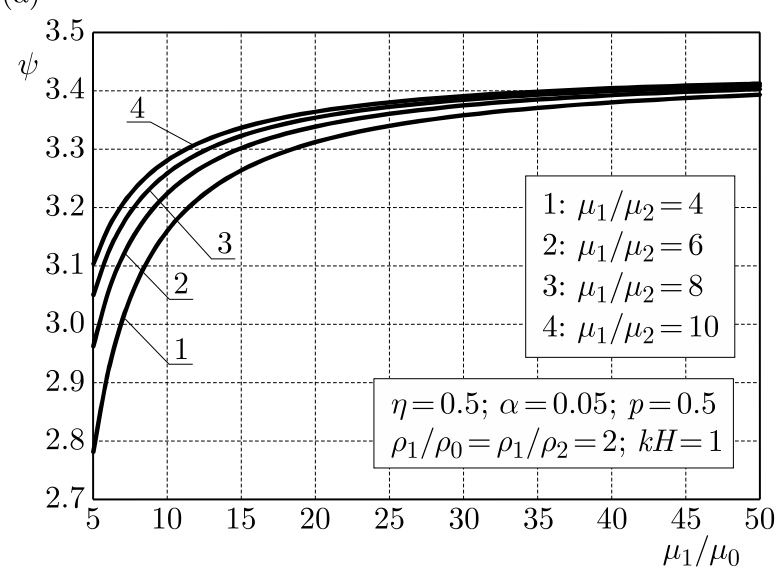

(b)

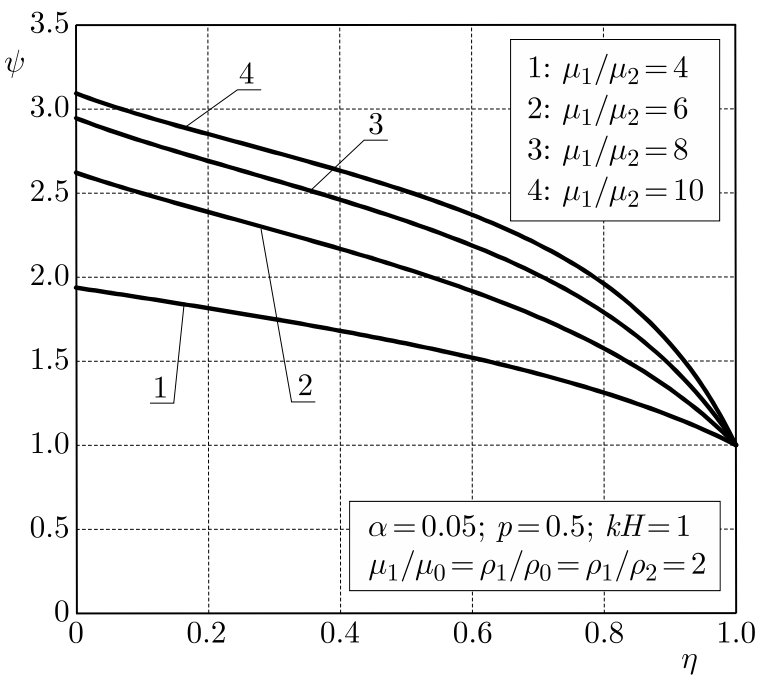

Fig. 4. The distributions of $\psi=c^{2} / c_{0}^{2}$ : (a) as a function of $\mu_{1} / \mu_{0}$, (b) as a function of $\eta$

The limit case $\eta \rightarrow 1$ leads to the homogeneous foundation with the shear modulus $\mu_{1}=2 \mu_{0}$, coated by the FGM layer with the shear modulus and the mass density dependent in the form given by (2.1) with respect to the distance from its lower boundary plane. In the case $\eta \rightarrow 0$, the half-space being the foundation with the shear modulus $\mu_{2}$ is obtained. The values of $\mu_{2}$ depend on the taken into account value of the ratio $\mu_{1} / \mu_{2}$. From the assumptions in Fig. $4 \mathrm{~b}$, it follows that the curves are adequate for the cases: curve 1 for $\mu_{0}=2 \mu_{2}$, curve 2 for $\mu_{0}=3 \mu_{2}$, curve 3 for $\mu_{0}=4 \mu_{2}$ and curve 4 for $\mu_{0}=5 \mu_{2}$, respectively. From Fig. $4 \mathrm{~b}$ it can seen that the values of $\psi$ decrease with an increase in the ratio $\mu_{1} / \mu_{2}$ for fixed values of the parameter $\eta$.

\section{Final remarks}

The problem of SH wave propagation in an elastic nonhomogeneous half-space is considered. The body is assumed to be composed of the FGM layer being a coating and periodically stratified two-layer half-space. The investigations are limited to the anti-plane shear harmonic waves in the nonhomoeneous body on the assumption that the boundary surface is free of loadings. The main aim is to determine the wave speed by using the wave number and the mechanical properties of the components of the half-space. The numerical results present the wave speed in the dimensionless form. The obtained figures show the influence of the nonhomogeneity of the coating layer as well as the nonhomogenity of the foundation on the wave speed. The assumptions of $p=0, \mu_{1}=\mu_{2}=\mu_{0}, \rho_{1}=\rho_{2}=\rho_{0}$ lead to Love's wave propagation in the homogenous halfspace coated by the homogeneous layer well-known in the literature (see for example Achenbach, 1973; Nowacki, 1970), which is shown in Appendix.

\section{A. Appendix}

Taking into account

$$
p=0 \quad \mu_{1}=\mu_{2} \quad \rho_{1}=\rho_{2}
$$


and using equation $(2.22),(2,23),(2.9)$ and $(2.13)$, it follows that

$$
C=\mu_{1} \quad q=k \sqrt{\frac{c^{2}}{c_{0}^{2}}-1} \quad \beta=k \sqrt{1-\frac{c^{2}}{c_{1}^{2}}} \quad c_{1}^{2}=\frac{\mu_{1}}{\rho_{1}} \quad c_{0}<c<c_{1}
$$

Substituting (A.1) and (A.2) into (2.29) and using following relations (Lebiediev, 1957)

$$
\begin{aligned}
J_{\frac{1}{2}}(z) & =\sqrt{\frac{2}{\pi z}} \sin z & J_{-\frac{1}{2}}(z) & =\sqrt{\frac{2}{\pi z}} \cos z \\
Y_{\frac{1}{2}}(z) & =-J_{-\frac{1}{2}}(z) & Y_{-\frac{1}{2}}(z) & =J_{\frac{1}{2}}(z)
\end{aligned}
$$

we obtain

$$
\begin{aligned}
\mu_{0} q & {\left[\sin \frac{q}{\alpha} \cos \left(q\left(\frac{1}{\alpha}+H\right)\right)-\cos \frac{q}{\alpha} \sin \left(q\left(\frac{1}{\alpha}+H\right)\right)\right] } \\
& +\mu_{1} \beta\left[\cos \frac{q}{\alpha} \cos \left(q\left(\frac{1}{\alpha}+H\right)\right)+\sin \frac{q}{\alpha} \sin \left(q\left(\frac{1}{\alpha}+H\right)\right)\right]=0
\end{aligned}
$$

From equation (A.4), it follows that

$$
\mu_{1} \beta=\mu_{0} q \tan (q H)
$$

Equation (A.5) agrees with the characteristic equation for the case of Love's wave presented in the monograph by Nowacki (1970) (p. 612, eq. (13)).

\section{References}

1. Achenbach J.D., 1973, Wave Propagation in Elastic Solids, North-Holland Publ. Co., Amsterdam-London

2. Achenbach J.D., Balogun O., 2010, Anti-plane surface waves on a half-space with depthdependent properties, Wave Motion, 47, 59-65

3. Alenitsyn A.G., 1964, Rayleigh waves in a nonhomogeneous elastic slab, Prikladnaya Matematika i Mekhanika, 28, 5, 880-888

4. Alshits V.I., Maugin G.A., 2005, Dynamics of multilayers: elastic waves in an anisotropic graded or stratified plate, Wave Motion, 41, 357-394

5. Bielski W.R., Matysiak S.J., 1992, Surface waves in a periodic two-layered elastic half-space, Acta Mechanica, 91, 47-55

6. Birykov S.V., Gulyaev Yu.V., Krylov V.V., Plessky V.V., 1995, Surface Acoustic Waves in Inhomogeneous Media, Springer, Berlin

7. Brekhovskikh L.M., 1960, Waves in Layered Media, Academic Press, New York

8. Calladine C.R., Greenwood J.A., 1978, Line and point loads on a non-homogeneous incompressible elastic half-space, Quarterly Journal of Mechanics and Applied Mathematics, 31, 507-529

9. Cerveny V., Popov M.M., Psencik I., 1982, Computation of wave fields in inhomogeneous media - Gaussian beam approach, Geophysical Journal of the Royal Astronomical Society, 70, $109-128$

10. Destrade M., 2007, Seismic Rayleigh waves on an exponentially graded, orthotropic elastic halfspace, Proceedings of the Royal Society of London. Series A, 463, 495-502

11. Kamke E., 1976, Handbook of Ordinary Differential Equations (in Russian), Science, Moscow 
12. Kennett B.L.N., 1983, Seismic Wave Propagation in Stratified Media, Cambridge University Press

13. Kulchytsky-Zhyailo R., Koøodziejczyk W., 2007, On axisymmetrical contact problem of pressure of rigid sphere into a periodically two-layered space, International Journal of Mechanical Science, 49, 704-711

14. Kulchytsky-Zhyhailo R., Matysiak S.J., 2005, On some heat conduction problem in a periodically two-layered body. Comperative results, International Communications in Heat and Mass Transfer, 32, 332-340

15. Kulchytsky-Zhyhailo R., Matysiak S.J., 2006, On distributions in a semi-infinite periodically stratified layer, Bulletin of the Polish Academy of Science, Technical Sciences, 54, 45-49

16. Kulchytsky-Zhyhailo R., Matysiak S.J., Perkowski D.M., 2006, On displacements and stresses in a semi-infinite laminated layer. Comperative results, Meccanica, 42, 117-126

17. Lebiediev N.N., 1957, Special Functions and their Applications (in Polish), PWN, Warsaw

18. MATYSiAK S.J., 1996, On the microlocal method in modeling of periodically layered composites, Journal of Theoretical and Applied Mechanics, 33, 481-487

19. Matysiak S.J., Mieszkowski R., Perkowski D.M., 2009, Surface waves in a periodic half-space with the boundary normal to the layering, Acta Mechanica, 207, 235-243

20. Matysiak S.J., Woźniak C., 1987, Micromorphic effects in a modelling of periodic multilayered elastic composites, International Journal of Engineering Sciences, 25, 549-559

21. MATYSiAK S.J., Woźniak C., 1988, On the microlocal modelling of thermoelastic composites, Journal of Technology Physics, 29, 85-97

22. Nayfen A.H., 1995, Wave Propagation in Layered Anisotropic Media, North-Holland, Amsterdam

23. Nowacki W., 1970, Theory of Elasticity (in Polish), PWN, Warsaw

24. Shuvalov A.L., Poncelet O., Kiselev A.P., 2008, Shear horizontal waves in transversely inhomogeneous plates, Wave Motion, 45, 605-615

25. Upadhyay S.K., Gupta O.P., 1972, SH-wave propagation in anisotropic inhomogeneous crustal layer, Pure and Applied Geophysics, 95, 67-73

26. Wang C.D., Tzeng C.S., Pan E., Liao J.J., 2003, Displacements and stresses due to a vertical point load in an inhomogeneous transversally isotropic half-space, International Journal of Rock Mechanics and Mining Sciences, 40, 667-685

27. Woźniak C., Woźniak M., 1995, Modeling of Composites. Theory and Applications (in Polish), IFTR Reports, Warsaw

28. RetTos C., 1990, In-plane vibrations of soil deposits with variable shear modulus. I. Surface wave, International Journal for Numerical and Analytical Methods in Geomechanics, 14, 209-222 